\title{
Olivocochlear Efferent Control in Sound Localization and Experience-Dependent Learning
}

\author{
Samuel Irving, ${ }^{1}$ David R. Moore, ${ }^{1}$ M. Charles Liberman, ${ }^{2}$ and Christian J. Sumner ${ }^{1}$ \\ ${ }^{1}$ Medical Research Council Institute of Hearing Research, Nottingham NG7 2RD, United Kingdom, and 2Department of Otology and Laryngology, Harvard \\ Medical School and Eaton-Peabody Laboratory, Massachusetts Eye \& Ear Infirmary, Boston, Massachusetts 02114-3096
}

Efferent auditory pathways have been implicated in sound localization and its plasticity. We examined the role of the olivocochlear system (OC) in horizontal sound localization by the ferret and in localization learning following unilateral earplugging. Under anesthesia, adult ferrets underwent olivocochlear bundle section at the floor of the fourth ventricle, either at the midline or laterally (left). Lesioned and control animals were trained to localize $1 \mathrm{~s}$ and $40 \mathrm{~ms}$ amplitude-roved broadband noise stimuli from one of 12 loudspeakers. Neither type of lesion affected normal localization accuracy. All ferrets then received a left earplug and were tested and trained over $10 \mathrm{~d}$. The plug profoundly disrupted localization. Ferrets in the control and lateral lesion groups improved significantly during subsequent training on the $1 \mathrm{~s}$ stimulus. No improvement (learning) occurred in the midline lesion group. Markedly poorer performance and failure to learn was observed with the $40 \mathrm{~ms}$ stimulus in all groups. Plug removal resulted in a rapid resumption of normal localization in all animals. Insertion of a subsequent plug in the right ear produced similar results to left earplugging. Learning in the lateral lesion group was independent of the side of the lesion relative to the earplug. Lesions in all reported cases were verified histologically. The results suggest the $0 \mathrm{C}$ system is not needed for accurate localization, but that it is involved in relearning localization during unilateral conductive hearing loss.

\section{Introduction}

Sound localization requires precise analysis of monaural spectral cues (produced by pinna shape) and of interaural differences in sound timing and intensity (produced by interaural distance and the head's acoustic shadow). Hearing loss can disrupt some or all these cues, yet ferrets with a unilateral earplug can adapt to localize accurately in both azimuth and elevation (King et al., 2000). Although this plasticity is rapid and complete in young animals, adult ferrets can also, with training, relearn to localize with an earplug (Kacelnik et al., 2006). Binocular eyelid suture does not affect the rate of improvement; thus visual feedback is not required, in contrast to the barn owl (Knudsen et al., 1991). After plug removal, performance returns to normal within a day, suggesting that neural rewiring has not occurred (Kacelnik et al., 2006). Ablation of primary auditory cortex (Nodal et al., 2010) reduces the rate and degree of localization improvement during earplugging, but lesioning the descending neural pathway from cortex to inferior colliculus prevents such improvements altogether (Bajo et al., 2010).

Descending neural circuits are prominent in the auditory system, including multisynaptic pathways from cortex to inner ear. The final link in this descending pathway, the olivocochlear (OC) system, projects from the superior olivary complex in the brainstem to the cochlea (Guinan, 2006). A role for OC feedback in

Received May 19, 2010; revised Nov. 3, 2010; accepted Nov. 7, 2010.

Research was supported by the Medical Research Council and by the National Institute on Deafness and Other Communication Disorders (Grants R01 DC 0188 and P30 05209 to M.C.L.).

Correspondence should be addressed to Dr. Christian J. Sumner, MRC Institute of Hearing Research, University Park, Nottingham NG7 2RD, UK. E-mail: chris@ihr.mrc.ac.uk.

DOI:10.1523/JNEUROSCI.2679-10.2011

Copyright $\odot 2011$ the authors $\quad 0270-6474 / 11 / 312493-09 \$ 15.00 / 0$ sound localization has been proposed based on indirect evidence: (1) the superior olivary complex is also where ascending neural circuits analyze interaural timing and intensity cues (Yin and Kuwada, 2010); (2) one component, the medial OC (MOC) system, provides subsecond, binaurally activated sound-evoked, negative feedback to the outer hair cells that decreases cochlear afferent responses (Maison and Liberman, 2000); and (3) the other component, the lateral OC (LOC) system, projects to afferent dendrites contacting inner hair cells, producing slow (minutes) excitatory and inhibitory feedback. LOC disruption unbalances the excitability of the cochlear nerves at the two ears, presumably affecting binaural sound intensity cues (Darrow et al., 2006). The OC system thus forms binaural feedback loops between the cochlea and the brainstem. These might function locally, or via descending projections from the inferior colliculus (Rajan, 1990; Groff and Liberman, 2003) or direct cortico-olivary projections (Xiao and Suga, 2002).

To date, only one study has directly examined the role of the OC system in spatial hearing. May et al. (2004) studied elevation discrimination in cats with the OC system cut at the midline, severing ipsilaterally responsive MOC fibers, but sparing the LOC and the contralaterally responsive MOC fibers. No deficit was observed when localizing noise bursts in quiet, perhaps because elevation judgments rely more on monaural spectral cues than on binaural cues (Van Wanrooij and Van Opstal, 2005). The aims of the present study were to examine the role of the OC system in azimuthal localization and in auditory plasticity following unilateral conductive hearing loss. We compared effects of a midline lesion with those from a laterally placed lesion, designed to sever unilaterally all fibers from both LOC and MOC systems, and unoperated controls. 


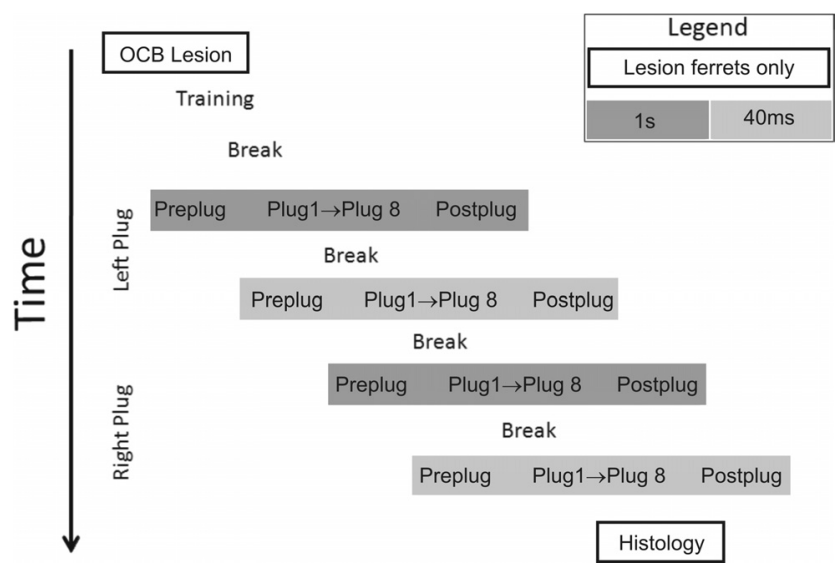

Figure 1. Schedule of the experiment. All groups were trained in the localization task, following recovery for lesioned animals. Once trained to criterion, all animals went through the same testing routine, as shown. Each experimental run was followed by a break period. After the final session, lesion animals were killed and the lesions were histologically verified.

\section{Materials and Methods}

Animals. Nine pigmented adult ferrets (5 male, 4 female) with clean ears were divided into three groups. Control animals $(n=4)$ included 3 unoperated animals and one animal with an unsuccessful OC lesion (see Histological analysis). Two lesion types were performed: (1) lateral lesion $(n=3)$, with an incision near the sulcus limitans ( 2 on the left, 1 on the right. The failed OC lesion included in the control group received a left incision), interrupting both MOC and LOC systems to the ear ipsilateral to the cut; and (2) midline lesion ( $n=2$ ), designed to remove MOC fibers to both ears that are driven by ipsilateral sound.

All animals were housed singly in rack-mounted custom built cages, exceeding UK Home Office regulations. Cages were populated with environmental enrichment measures (hammocks, balls, houses etc.) which were varied regularly, and all animals received daily exercise and play outside of their home cage, usually with other ferrets.

During training (Fig. 1), ferrets were deprived of fluids in their home cage, and trained to obtain water in a positive reinforcement conditioning behavioral procedure. At the end of each working day, animals were generally given a wet-food alternative to their normal diet, composed of protein pellets ground up with a food supplement (Cimicat, PetLife International Ltd.) and $\sim 5 \mathrm{ml}$ of water. Water regulation periods lasted 10-14 consecutive days before free access was returned for at least $4 \mathrm{~d}$. Animals were weighed and their general health was monitored daily, both during regulated and nonregulated periods. Regulation ceased if weight dropped $>20 \%$, or 2 SDs of their individual natural variation in weight, below the starting weight. All procedures were performed under license from the UK Home Office.

Apparatus and stimuli. Animals were tested in a circular enclosure (150 $\mathrm{cm}$ diameter) inside a double-walled sound-attenuated room (Industrial Acoustics Company), the inner walls of which were lined with $5 \mathrm{~cm}$ of mineral wool to reduce acoustic reflections. The walls and lid of the enclosure were constructed from wire mesh, and the floor was uncovered PVC. Twelve loudspeaker units were situated at $30^{\circ}$ intervals around the outside of the enclosure. Each custom-made unit comprised a speaker (Visatron FX10) and a solenoid-operated water spout, protruding to the inside of the enclosure, with an embedded, calibrated, infrared lick detector. Peripheral loudspeakers were concealed from view by opaque (but acoustically transparent) net fabric. Located in the center of the enclosure was a slightly raised "start position" platform with wire-frame barriers on the lateral and frontal sides as well as an infrared beam across the width of the start position, which was broken by the ferret's body when in the correct position. At the front of the platform was another "central" water spout. Delivery of sound and the detection of licks was driven by a MOTU 24 I/O system (Mark of the Unicorn), and the delivery of calibrated water rewards was performed via a USB interface. The hardware was controlled by custom software that also controlled and recorded the behavior. A closed-circuit video camera gave a live feed of the sound booth, allowing behavioral monitoring. Stimuli were amplitude-roved (55-75 dB SPL, $1 \mathrm{~dB}$ steps, in pseudorandom order) broadband noise bursts ( $1 \mathrm{~s}$ or $40 \mathrm{~ms}$ ), sampled at $96 \mathrm{kHz}$ and 16 bit resolution, and presented from any of the 12 loudspeakers in pseudorandom order. The bandwidth of the noise bursts was limited to $80-22,000 \mathrm{~Hz}$ by the response of the speaker (flat $\pm 6 \mathrm{~dB}$ in this range). The noise was gated on and off by a $2.5 \mathrm{~ms}$ raised cosine function.

Surgery and ear manipulations. Surgical levels of anesthesia were induced using intramuscular injections of medetomidine hydrochloride (Domitor, $0.1 \mathrm{mg} / \mathrm{kg}$; Pfizer) and ketamine (Ketaset, $5 \mathrm{mg} / \mathrm{kg}$, Fort Dodge Animal Health). Animals were intubated and anesthesia was maintained with $2-3 \%$ gaseous isoflurane. Core temperature was monitored with a rectal probe and maintained in the range $37-38^{\circ} \mathrm{C}$ with a thermostatically controlled heating blanket. Respiratory function was monitored via end-tidal $\mathrm{CO}_{2}$ and heart rate was monitored, using a pair of subdermal electrodes on either side of the thorax. The head was clamped firmly by the nose and mouth. All surgery was conducted under aseptic conditions.

The OC bundle was interrupted with a surgical microknife via a small rostrocaudal incision into the dorsal surface of the brainstem, as diagrammed in Figure $2 \mathrm{~A}$. The posterior and dorsal aspect of the skull was exposed and a craniotomy was made into the posterior fossa by removing the occipital bone between the foramen magnum and the nuchal crest. After opening the dura, the cerebellum was gently elevated to allow access to the floor of the fourth ventricle. Using surface landmarks, the OC bundle was cut using a microsurgery knife at the level of the sulcus limitans (lateral lesion, Fig. $2 \mathrm{~A}$ ) or along the sagittal plane (midline lesion). The craniotomy was covered with Gelfoam (Pfizer,) before the muscles and skin were repositioned and sutured. The animals were placed in a recovery cage on a temperature-regulated heat mat until they were mobile and put back in their cage. Then, $10 \mathrm{ml}$ of saline was administered subcutaneously to replace fluids, and analgesia was given for the next $7 \mathrm{~d}$. Recovering animals were monitored for weight, temperature, and food and fluid intake, and closely monitored for signs of distress in accordance with the UK Home Office recovery guidelines.

Earplugs were E.A.R. Classic foam plugs, cut to fit inside a ferret's ear. Earplugs were placed initially in the left ear (with the exception of the right-lateral lesion animal, which was first plugged in the right ear) and subsequently the right ear (Fig. 1). Under sedation with medetomidine hydrochloride (Domitor, $0.1 \mathrm{mg} / \mathrm{kg}$; Pfizer), the ear canal was checked for infection and wax build-up. If clean, the earplug was inserted and left to expand for a few minutes before being checked to confirm that the canal was properly occluded. The meatus and concha were then filled with ear mold impression compound (Otoform-K2, Dreve Otoplastik). This type of earplug provides attenuation of $\sim 40 \mathrm{~dB}$ from $0.5-16 \mathrm{kHz}$ (Hine et al., 1994; Moore et al., 1999).

Behavioral task and training. The task was closely analogous to that presented in detail previously (Moore et al., 1999; King et al., 2001; Kacelnik et al., 2006; Nodal et al., 2010). Briefly, ferrets first underwent shaping: they were required to assume the correct position on the central platform, with their body straight and face forwards, and to lick the center spout. The center spout then produced a reward, of 10 drops (15 $\mu \mathrm{l} / \mathrm{drop}$ ) of water. In the next stage, licking the center spout resulted in the presentation of a continuous noise burst from one of the loudspeakers. The sound began 500-2000 ms after first licking (random delay). Ferrets, out of natural curiosity, approached the target loudspeaker. Licking a water spout located immediately above the target loudspeaker terminated the stimulus and resulted in the delivery of a 10 drop water reward. Ferrets quickly (usually within one session) learned to approach the source location of long sounds and lick the spouts to receive water. Incorrect responses were unrewarded and initiated a "correction trial" where the stimulus was repeated from the same loudspeaker: it had to respond correctly to every stimulus location before it could receive a reward. Correction trials were omitted from the data analysis.

Animals were trained twice daily in sessions of 20-60 min, until they were satiated. After a few sessions, the animal responded correctly to $>90 \%$ of the stimuli. Once this was achieved for two consecutive runs, the task was made progressively more difficult. Initially, the center re- 

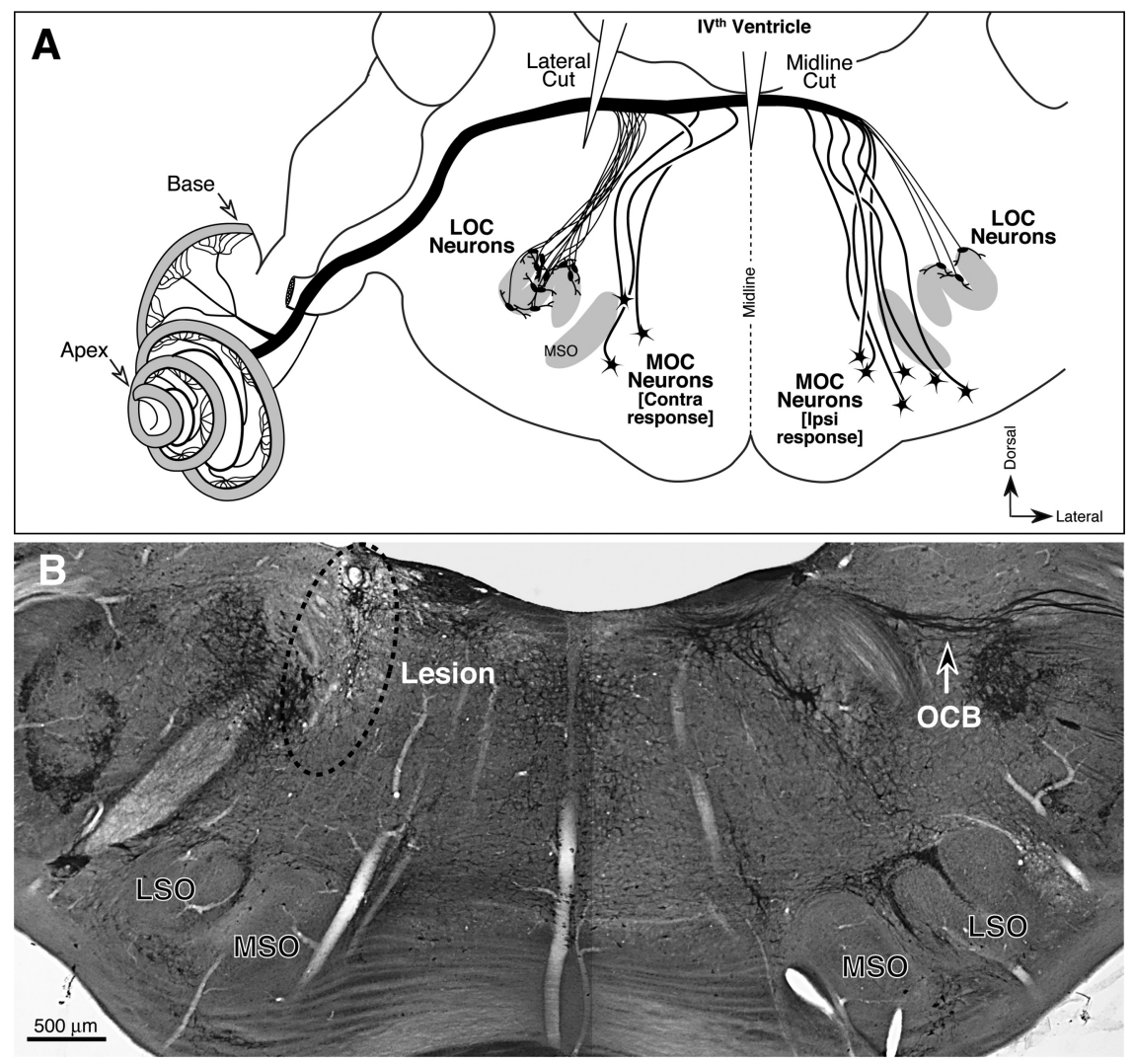

Figure 2. $A$, Schematic showing the origins of $M O C$ and $L O C$ fibers in the superior olivary complex, the relative numbers from the ipsilateral and contralateral sides of the brainstem, and the locations of the two types of $O C$ lesions used in the present study. $\boldsymbol{B}$ Coronal brainstem section stained for $\mathrm{AChE}$, showing a successful lateral lesion. Arrow at right indicates the fibers of the 0 C bundle. On the corresponding region of the left side, there is an obvious scar (dotted ellipse) where the microknife cut into the floor of the IVth ventricle. MSO, Medial superior olive; LSO, lateral superior olive.

ward was reduced from 10 drops to 1 , then the probability of getting 1 drop in the center was reduced from 1 to 0.1 . Subsequent changes were to reduce the stimulus duration, waiting for performance to stabilize at each duration. Animals were considered trained when they could consistently localize a $1 \mathrm{~s}$ noise burst with an accuracy $>90 \%$. One animal with a lateral lesion, additional to those listed above, did not reach this criterion and was omitted from analysis. Once trained to criterion at $1 \mathrm{~s}$, the duration was systematically reduced to $40 \mathrm{~ms}$ and training continued until performance was stable. Training time varied between individuals, but there was no systematic difference between the training times for lesion and control animals.

After $2 \mathrm{~d}$ ( 4 sessions) of preplug testing (Fig. 1; $1 \mathrm{~s}$ stimulus), a plug was inserted in the left ear (right ear for the animal with the lateral lesion on the right). Performance was then measured for $10 \mathrm{~d}$, at the end of which the plug was removed, the ear was checked for infection and wax, and the animal was tested for 4 more sessions to determine postplug performance. Following a break ( $\geq 4 \mathrm{~d}$ ), this protocol was repeated for the 40 $\mathrm{ms}$ stimulus, then with the plug in the other ear at $1 \mathrm{~s}$ and then $40 \mathrm{~ms}$ stimulus durations (Fig. 1).

Histological processing and lesion verification. Animals were killed with a large dose $(\sim 3 \mathrm{ml})$ of Euthanal and perfused transcardially with $4 \%$ paraformaldehyde. Following a further 3-4 h in fixative, brainstems were placed in sucrose/fixative (for cryoprotection) and cochleae in EDTA (to decalcify). Brainstems were sectioned at $40-80 \mu \mathrm{m}$ on a freezing microtome and stained for acetylcholinesterase (AChE). Whole mounts of the cochleae were processed immunohistochemically with antineurofilament (to mark all fibers, both afferent and efferent) and antisynaptophysin (to mark efferent terminals). To control for staining variability, the two cochleae from each case were processed together. AChE-stained brainstems were examined using bright-field microscopy, and cochleae were examined using a confocal microscope.
For each case, cochlear lengths of all dissected pieces were measured with a custom Image plugin, and the cochlear locations corresponding to the $25 \%, 50 \%$ and $75 \%$ locations along the cochlear spiral identified. At each of these locations, a confocal $z$-stack was obtained, using a $20 \times$ oil-immersion objective. Each resultant image contained $0.75 \mathrm{~mm}$ of cochlear length and spanned the entire height of the organ of Corti in the $z$-dimension. These image stacks were ported to Amira (Visage Imaging Software) for 3D quantification of the volume of synaptophysin-positive terminals in the IHC and $\mathrm{OHC}$ areas, using criterion values for pixel intensity of 60-70, depending on the overall brightness of staining: the same value was always used for both ears from each animal. For quantification, each image stack was cropped from 2048 to 1536 pixels in the " $x$ " dimension, and centered on the $x$-axis. The " $y$ " dimension was cropped to include only LOC or MOC terminals, as appropriate.

Data analysis. The $14 \mathrm{~d}$ experimental period was divided into preplug, plugged and postplug sections. The plugged section was further divided into 8 blocks, configured to ensure a similar number of trials within each block (generally $\sim 200$ ). Performance was measured and is illustrated using the "centre of mass" (CoM) method. Details of the CoM rationale and analysis, and an application to human listeners, are the subject of another article (Edmondson-Jones et al., 2010). This method, described briefly here, allows us to consider the proportion, direction, and size of errors simultaneously. It takes account of the circular nature of the responses, and also allows multivariate analyses of variance (MANOVAs) to be applied to the entire dataset.

In the CoM analysis, the test arena has a radius of 1 . Cartesian axes (with origins at the circle's center) provide an $(x, y)$ coordinate for each point in the arena. If the stimulus and response arrays are rotated, so that a given stimulus speaker is always at $0^{\circ}$, the CoM analysis gives equal weight to each response location associated with that stimulus. In this way, if all responses are correct, the CoM mean will have the coordinates $(0,1)$. For incorrect responses, the $x$ and $y$ values change in relation to the size of the error. For example, if 10 stimuli are played, with 5 responses correct, and 5 erroneous, but all erroneous responses are made to the same speaker location, the CoM measurement will be a coordinate situated midway along the line connecting the two response speakers. Larger errors place the CoM further toward the origin of the plot. Throughout the paper, comparisons of accuracy and error refer to differences in the CoM $(x, y)$ coordinates toward or away from the point of perfect sound localization $(0,1)$. For graphic representation, the stimulus-response locations are counter-rotated. Data points arrayed around the perimeter indicate correct responses or errors at nearby speakers, whereas points gravitating toward the origin indicate errors toward far away speakers. Percentage correct is also illustrated with radar plots for qualitative comparisons with previous studies of ferret localization (Kacelnik et al., 2006).

CoM values were analyzed statistically (in SPSS) using MANOVAs applied on a trial-by-trial basis, to test specific hypotheses. For a given experimental group, we ran separate MANOVAs to compare CoMs across sessions or within a single group of sessions. Individual animals and target speaker location (expressed either as angle, or hemifield, which excluded speakers at 0 and $180^{\circ}$ ) were usually included as independent variables. The degrees of freedom varied with the independent variables and the numbers of trials available for the test. Further MANOVAs 
were run to compare performance between the experimental groups, or the side of the earplug.

We also recorded and analyzed one other measure of performance: the time between the onset of the stimulus and the time when the ferrets first licked a response spout, located directly above one of the loudspeakers. This is a crude measure of reaction time, and is usually longer for incorrect responses (Nodal et al., 2008). It also offers a metric of mobility, which is useful when assessing the performance of operated animals (Bajo et al., 2010).

\section{Results}

\section{Histological analysis}

Success of the OC lesions was assessed, first, by analysis of brainstem sections stained for acetylcholinesterase, which reveals cholinergic fiber tracts including the OC bundle. As schematized in Figure $2 A$, a correctly positioned lateral cut can eliminate both MOC and LOC systems to one ear. Based on the brainstem analysis, three of four laterally placed lesions transected most of the OC bundle on the side of the cut (Fig. 2B), whereas one missed the bundle completely. The brainstem analysis further confirmed that both of the midline lesions were correctly positioned to transect most of the crossing OC fibers to both ears. A correctly positioned midline cut (Fig. 2A) should eliminate the majority of MOC fibers to both ears, while sparing the majority of LOC fibers.

The degree of cochlear de-efferentation was further estimated by examination of cochlear whole mounts immunostained for an efferent marker (synaptophysin) and a neuronal marker (neurofilament). As shown in Figure 3, the density of efferent innervation in the normal ferret ear varies with cochlear location, as described for other mid-frequency mammals (e.g., cat) (Liberman et al., 1990). The MOC innervation to the outer hair cell area is most profuse in the upper basal turn (25\% of the distance from the base), where each outer hair cell is contacted by several MOC terminals. MOC innervation density has fallen dramatically by the $75 \%$ locus, where terminals are largely restricted to the first row of outer hair cells. The density of LOC innervation, to the cochlear nerve terminals in the inner hair cell area, varies less dramatically along the cochlear spiral.

The cochlear analysis of de-efferentation was consistent with the conclusions from the brainstem sections: the images in Figure $4 A-C$ show the upper basal turn (25\% locus); similar results were seen in the $50 \%$ and $75 \%$ loci. Both MOC and LOC systems were intact on both sides of the case in which the lateral lesion missed the OC bundle (top row of images). All three cases with "successful" lateral lesions showed a patchy loss of LOC and MOC fibers, of varying degree, ipsilateral to the cut, and normal innervation on the contralateral side (Fig. $4 B$ ). The quantitative analysis of LOC and MOC terminal densities, shown in Figure 4, $D$ and $E$, respectively, suggest that the loss of LOC fibers was $>85 \%$ and the loss of MOC fibers was $>70 \%$, in all three lateral lesion cases, in all cochlear regions. The midline lesion (Fig. 4C) shows symmetrical and diffuse loss of MOC terminals on both sides: there also appears to be some loss of LOC terminals in the inner hair cell area. In the second midline lesion animal, the immunostaining was unsuccessful.

Based on this histological analysis, the animal with the unsuccessful lateral lesion was considered to have a normal efferent innervation. Since localization performance of this animal was comparable to that of the nonoperated animals, it was included with the control group.

\section{Effect of earplugging on sound localization}

Control animals were able to localize $1 \mathrm{~s}$ stimuli with a high level of accuracy (Fig. 5, preplug), as expected. The CoM analysis of the control, preplug data showed no overall difference in performance between individual animals, considering all target speaker locations $\left(F_{(6,1112)}=1.18 ; p=0.31\right)$ or between stimuli presented in the left and the right hemifields $\left(F_{(2,493)}=1.14 ; p=0.32\right)$. In the first block of trials after inserting a plug into the left ear, there was an immediate decrease in localization accuracy, relative to preplug performance and considering all speaker locations $\left(\mathrm{F}_{(2,1509)}=87.40 ; p<0.001\right)$ (Fig. 5, preplug vs plug 1 ). Comparing responses to stimuli in the left and right hemifields, this reduction in accuracy was more marked in the left, ipsilateral to the earplug, than in the right hemifield $\left(F_{(2,1317)}=20.88 ; p<\right.$ $0.001)$. Responses to front and rear speakers were shifted toward the lateral speaker at $90^{\circ}$, and accuracy was best in response to sounds from right and rear locations (e.g., $150^{\circ}$ ). In addition to a drop in performance, the time taken to approach the target spout was longer with a plug in place than before insertion (paired $t$ test, $p<0.001$ ), consistent with previous reports (Nodal et al., 2010). 

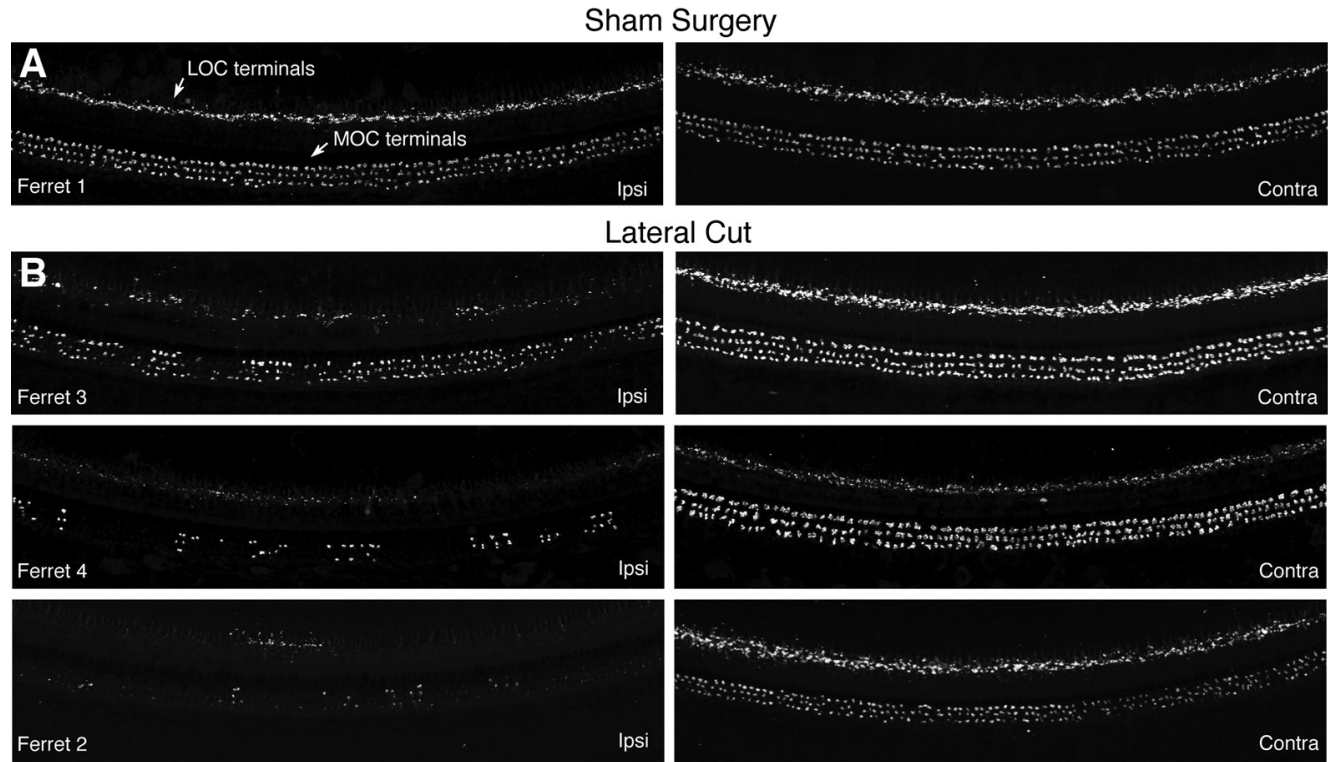

Midline Cut
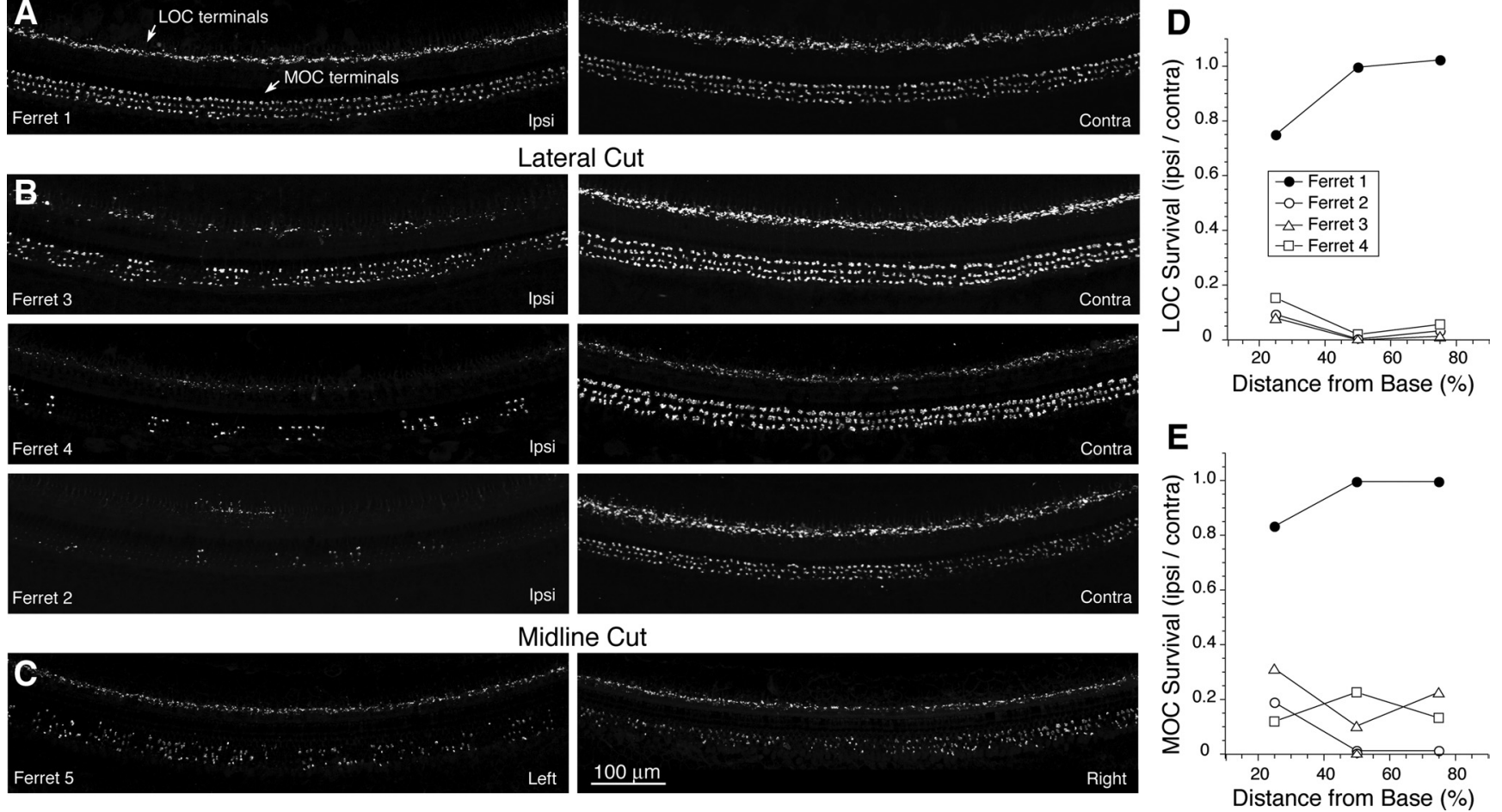

Figure 4. Degree of de-efferentation in each of the $0 C$ lesions cases. $A-C$, Place-matched images of cochlear whole mounts immunostained for synaptophysin. Each row of images shows cochlear regions $25 \%$ of the distance from the basal end: Ipsi denotes the ear on the same side as the lateral lesion; contra, the opposite. Each image is the maximum projection of a $z$-stack of confocal images spanning the entire height of the organ of Corti. Scale bar in Capplies to all images. D, E, Quantification of the degree of de-efferentation for the LOC system (D) or the MOC system $(\boldsymbol{E})$ in each of the cases with an attempted lateral lesion. Total volumes of all labeled terminals were measured in image stacks such as those pictured in $\boldsymbol{A}-\boldsymbol{C}$ : degree of de-efferentation was estimated by dividing the data from place matched image stacks from the two sides of each case.
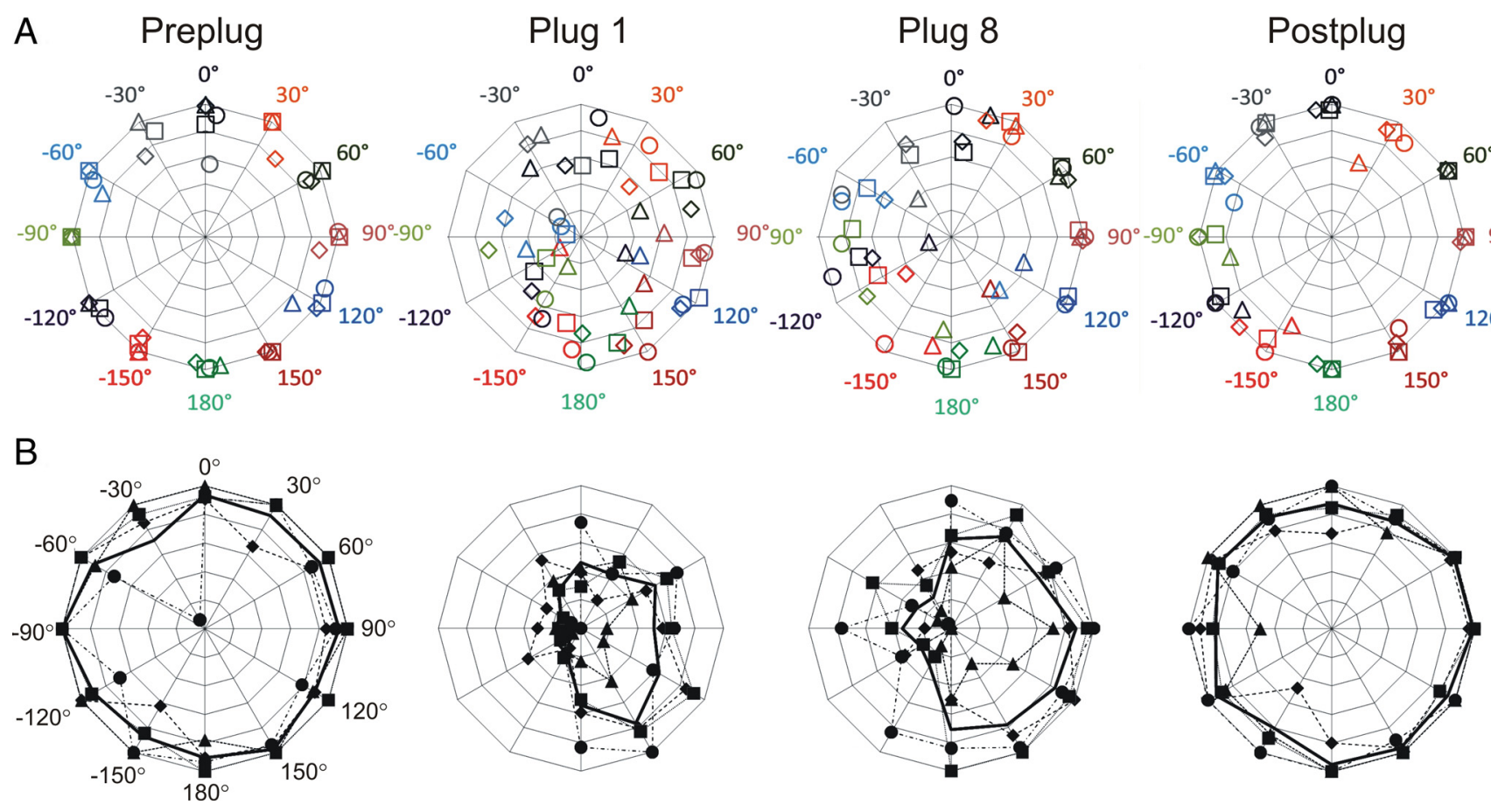

Figure 5. Sound localization by control animals before (preplug), during (plug 1, plug 8), and following (postplug) removal of the first, left earplug; $1 \mathrm{~s}$ stimulus duration. $\boldsymbol{A}$, CoM for each stimulus location (colors) for each animal (different symbols). $\boldsymbol{B}$, Radar plots showing the absolute accuracy for individual animals (dotted lines) and the mean (bold). 

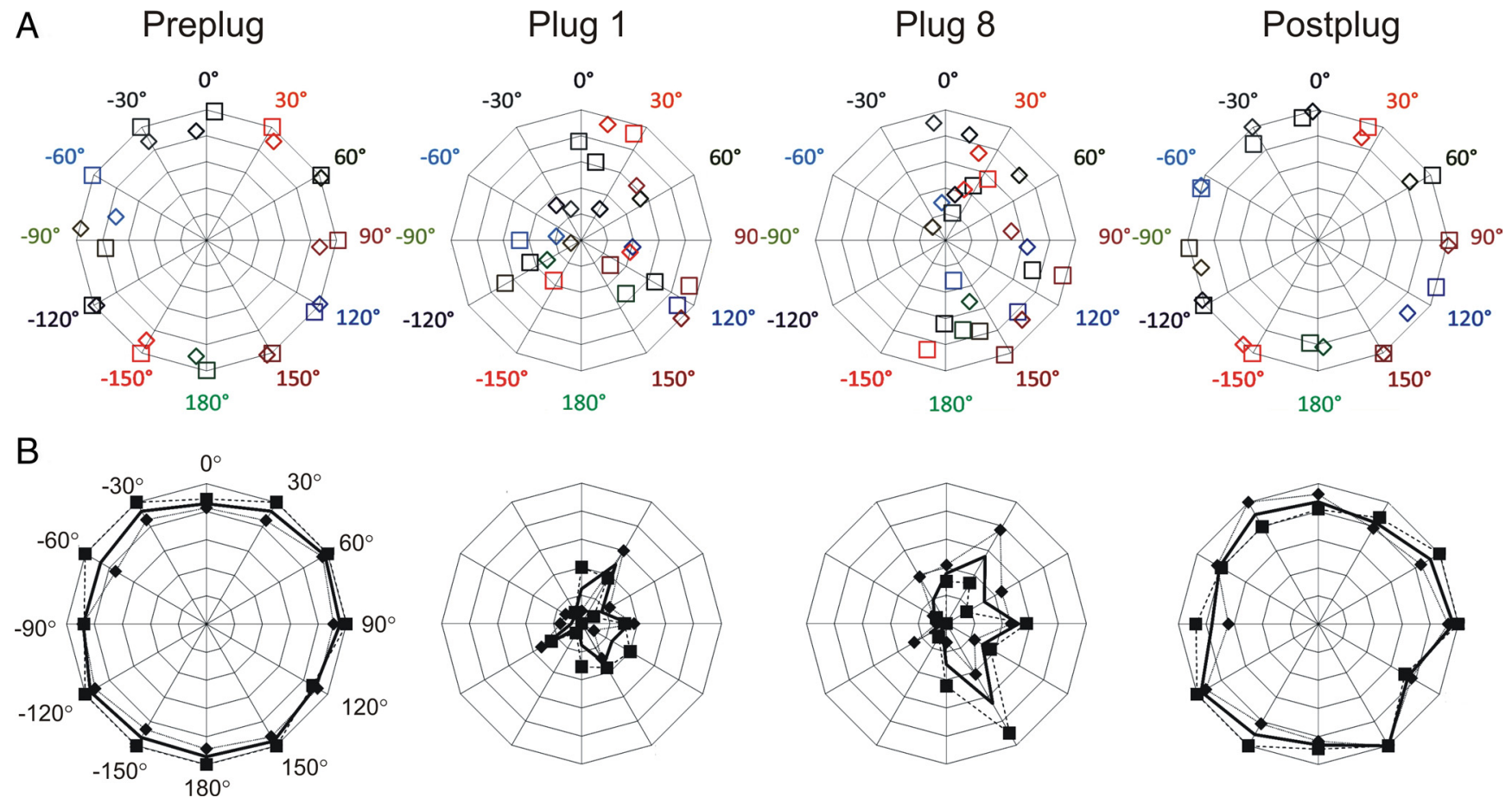

Figure 6. Localization by midline lesion animals. $\boldsymbol{A}$, CoM plots. $\boldsymbol{B}$, Radar plots. Details as in Figure 5 .

Training with the plug in place improved localization ability across the $10 \mathrm{~d}$ of earplugging $\left(F_{(14,14,090)}=4.14 ; p<0.001\right)$ (Fig. 5 , plug 1 vs plug 8 ), particularly in response to sounds originating from the right hemifield, contralateral to the earplug (speakers at $60^{\circ}$ to $150^{\circ}$ ). Although overall accuracy remained poor in response to sounds originating from the ipsilateral hemifield, there was a significant improvement with time $\left(F_{(14,12,282)}=3.51 ; p<\right.$ $0.001)$. The improvement across the plugged sessions was also significant in 3 of 4 individual ferrets (1-way MANOVA for each animal, $p<0.001$ ), including the ferret that received an unsuccessful lesion.

Removal of the left earplug (Fig. 5, postplug) improved performance in postplug trials relative to the last plugged session $\left(F_{(2,1584)}=58.26 ; p<0.001\right)$ (Fig. 5 , plug 8 vs postplug), and eliminated the performance difference between left and right hemifields $\left(F_{(2,664)}=0.68 ; p=0.51\right)$. Performance in the postplug sessions was comparable to that in the preplug sessions $\left(F_{(2,1313)}=0.73 ; p=0.49\right)$ (Fig. 5 , preplug vs postplug), showing that removing the plug rapidly and fully restored preplug performance. The major effects of earplugging on sound localization can also be seen in the more conventional measure of percentage correct (Fig. 5B), which does not account for the size of the errors. Subsequent insertion of a right earplug (Fig. 1) produced qualitatively and largely quantitatively the same trends as the initial, left earplug (see supplemental material, available at www.jneurosci.org, for details).

We also tested our ferrets when localizing 40 ms noise bursts. These data generally confirmed the preplug performance and immediate effects of earplugging. However, none of the groups showed significant learning with an earplug at $40 \mathrm{~ms}$, so no conclusions could be drawn about the effects of lesions on learning (see supplemental material, available at www.jneurosci.org, for details).

\section{Midline olivocochlear lesions}

Before earplug insertion, midline lesion animals learned to localize $1 \mathrm{~s}$ stimuli as accurately as control animals (Fig. $6 ; F_{(2,999)}=$ $0.30 ; p=0.74)$. Left plug insertion resulted in an immediate reduction in localization accuracy in both hemifields, relative to preplug performance $\left(F_{(2,543)}=66.684 ; p<0.001\right)$. The reduction was greater in the left (ipsilateral) hemifield, on the side of the plug, than in the right (contralateral) hemifield $\left(F_{(2,543)}=\right.$ $4.91 ; p<0.01)$. This decrease in performance was more pronounced than that seen in control animals $\left(F_{(2,1877)}=7.30\right.$; $p=0.001)$. Responses to stimuli originating from the left were shifted toward the right (unplugged) side, as for controls.

In contrast to controls, training of midline lesion animals with the left ear plugged did not lead to improved performance $\left(F_{(14,2966)}=1.05 ; p=0.40\right)$. We also tested for effects of training in individuals, and no individual in this group showed a significant improvement with training.

Removal of the plug caused an immediate improvement when compared with the last plugged session $\left(F_{(2,438)}=68.62 ; p<\right.$ $0.001)$, and postplug performance was comparable between the midline and control groups $\left(F_{(2,888)}=2.18 ; p=0.11\right)$. Postplug and preplug performance in the midline lesion animals did not $\operatorname{differ}\left(F_{(2,556)}=1.72 ; p=0.18\right)$ showing, as for controls, a rapid and full recovery.

Insertion of a right earplug had very similar effects to those seen for the left earplug (see supplemental material, available at www.jneurosci.org, for more details). Training did not produce a significant improvement in performance in the group $\left(F_{(14,2516)}=1.54 ; p=0.09\right)$, or in any individuals. One difference however, was that the immediate deficit on insertion of the plug was this time no worse than that seen in controls $\left(F_{(2,902)}=1.381 ; p=0.252\right)$.

The midline group were not significantly different from controls in the time they took to reach the target spout, before plug 

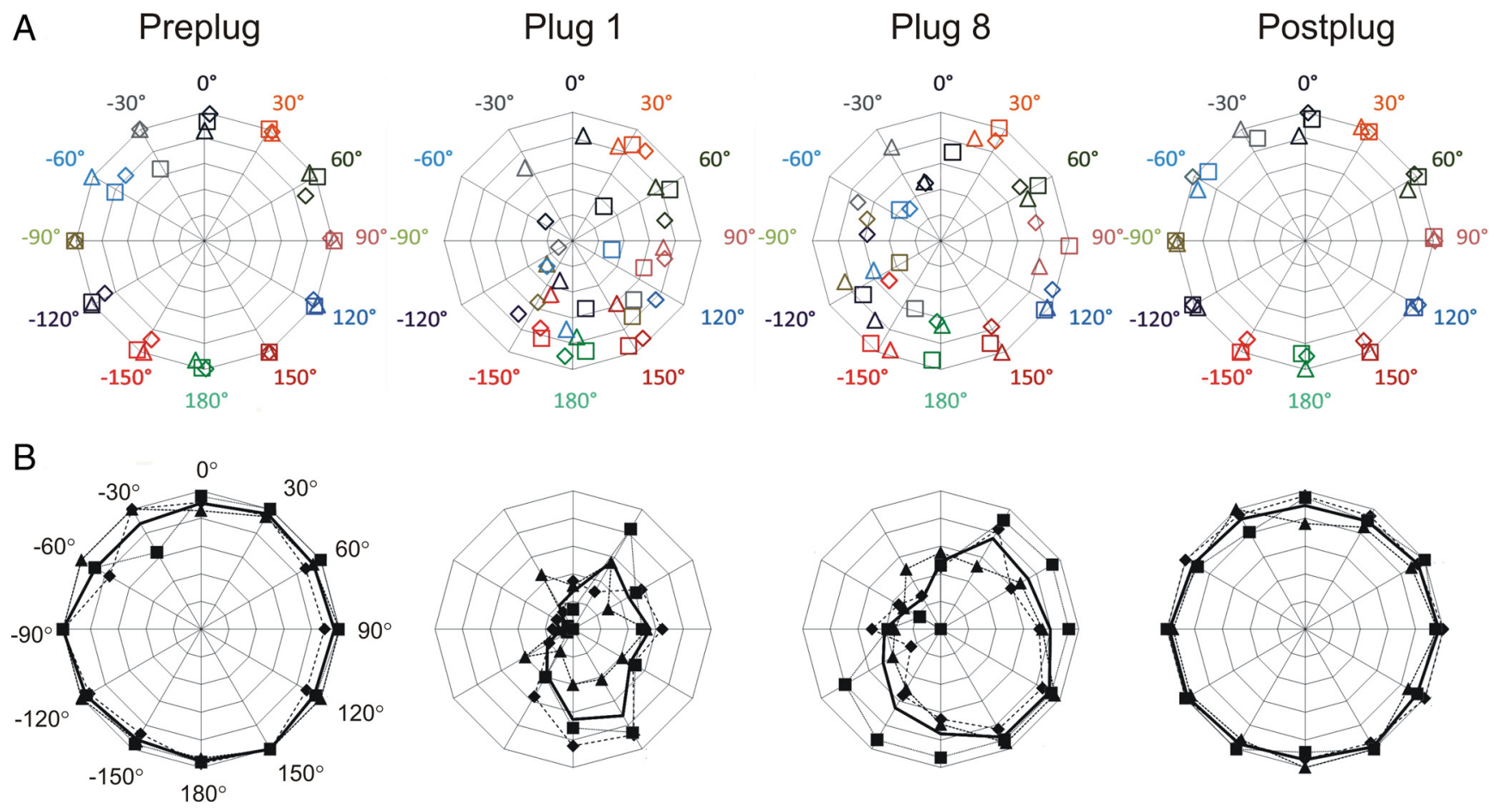

Figure 7. Localization by lateral lesion animals. $\boldsymbol{A}$, CoM plots. $\boldsymbol{B}$, Radar plots. Details as in Figure 5.

insertion (paired $t$ test, $p=0.31$; data not shown). This suggests that surgery had not affected mobility. As in the control group, the time taken to approach the target spout was longer with a plug in place than before or following insertion (paired $t$ tests, $p<$ 0.001 ). However, when plugged, midline lesion ferrets (unlike the lateral lesion group) took longer to respond than controls $(p<$ 0.001 ), either when considering all plugged sessions, or when considering only the first or last plugged session.

\section{Lateral olivocochlear lesions}

Preplug localization performance for $1 \mathrm{~s}$ stimuli by lateral lesion animals did not differ significantly from that of the control or the midline lesion groups (Fig. 7$)$, either in accuracy $\left(F_{(4,3406)}=0.93\right.$; $p=0.44$ ) or in the time to approach the target spouts (paired $t$ tests, $p>0.05$ ). Two ferrets in the lateral lesion group had the OC bundle sectioned on the left, and one (Fig. 7, diamond markers) had a section on the right. As localization performance at each stage of testing and training showed no systematic differences between these animals, when the data for the right lesioned ferret were reflected about the midline, data from this animal were incorporated with those of animals with left side lesions.

Insertion of an earplug impaired localization accuracy in the session immediately following insertion $\left(F_{(2,1348)}=197.68 ; p<\right.$ $0.001)$, to an extent comparable to that seen following midline lesions $\left(F_{(2,1697)}=2.37 ; p=0.09\right)$. This immediate deficit was more pronounced than that for controls $\left(F_{(2,2479)}=11.47 ; p<\right.$ $0.001)$. As in the other groups, performance was worse in response to stimuli originating from the side of the plug than from the opposite hemifield $\left(F_{(2,1141)}=73.15 ; p<0.001\right)$.

Training with the earplug in place improved localization $\left(F_{(14,9274)}=7.29 ; p<0.001\right)$. This improvement across the training period was not significantly different to controls $\left(F_{(14,21,714)}=1.69 ; p=0.05\right)$. Removal of the plug resulted in improved performance, compared with the last session with a plug $\left(F_{(2,1131)}=71.47 ; p<0.001\right)$. Performance did not differ significantly between the preplug and postplug sessions $\left(F_{(2,1174)}=0.83 ; p=0.44\right)$.

Insertion of the right earplug produced generally comparable consequences to those seen in the control group. These included learning, and this time the initial effect of the plug was no different to the controls. The largest deficits were always for targets on the side of the plugged ear. Thus the deficits mirrored those seen in the control group, and did not depend on whether the plug was ipsilateral or contralateral to the side of the lesion. Further details of right earplugging are given in the supplemental material (available at www.jneurosci.org).

\section{Discussion}

\section{Olivocochlear feedback control}

Hair cell systems receive feedback control directly from the brain, enabling considerable modulation of afferent input. However, little is known about the role this feedback plays in auditory perception. In mammals, neural control signals come from myelinated MOC fibers projecting to outer hair cells, and from unmyelinated LOC fibers projecting to dendrites of cochlear nerve (sensory) fibers near inner hair cells (for review, see Guinan, 1996). The MOC system is both sound evoked and frequency specific (Liberman and Brown, 1986). It decreases mechanical vibration in the cochlea by reducing gain in the cochlea's biological amplifiers. This feedback has a fast time constant $(\sim 100 \mathrm{~ms})$ (Liberman et al., 1996), and can enhance responses to acoustic transients by reducing responses to background noise (Winslow and Sachs, 1987). The LOC system has both excitatory and inhibitory components and a much slower time constant (tens of minutes) (Groff and Liberman, 2003). Single LOC neurons project to restricted regions of the cochlea (Brown, 1987), but it is unclear whether the LOC pathway responds to sound.

Both LOC and MOC systems originate from the superior olive (Warr and Guinan, 1979), a brainstem region involved in binaural processing and sound localization (Fig. 2A). MOC cells are 
monaural, receiving inputs from the contralateral cochlear nucleus. Two-thirds project back to the contralateral cochlea, thus forming a monaural feedback loop. The remainder project ipsilaterally (Schofield, 2010). Most LOC neurons project ipsilaterally, but receive binaural input. Removing the LOC system leads to an interaural imbalance in the responses of the cochlear nerve to equilevel sound (Darrow et al., 2006).

The OC system has the potential to modulate cues associated with location of sounds in space. However, there is little evidence that the feedback control is actually useful for localization. This study suggests, instead, the novel idea that one role of the OC system is to adjust the afferent signals from the two ears to allow the central processor to maintain accuracy in sound localization during acquired changes in hearing sensitivity, such as occur with unilateral conductive hearing loss (e.g., from middle ear infection).

\section{Olivocochlear control and sound localization with normal hearing}

The localization of sounds in space is based on a variety of acoustic cues. Elevation, in the sagittal plane, is cued predominately by sound spectrum, which differs with sound position because of the ridges and cavities within the external ear (May, 2010). Azimuthal position, in the horizontal plane, is normally cued by interaural level differences, produced by acoustic head shadowing and interaural timing differences introduced by the acoustic delay for off-midline sounds reaching the two ears (Yin and $\mathrm{Ku}-$ wada, 2010). Level differences are generally more prominent at high frequencies, because low frequencies diffract around the head, and timing differences are more important at low frequencies due to the longer period of sound. Prior study in cat (May et al., 2004) showed that localization performance on an elevation task with 200 ms noise bursts was not impaired by a midline OC lesion, which interrupts the ipsilaterally driven MOC fiber system to both ears. This is consistent with the idea that elevation is extracted from monaural spectral cues not requiring MOC modulation.

Here, we found no effect of either OC lesion on azimuthal localization performance, before insertion of the earplugs. It may be that sound localization normally does not require any "balancing" of the brain's binaural inputs. It is also possible that lowfrequency timing cues can be used for this task, as suggested by prior ferret studies (Kacelnik et al., 2006). Since the MOC system projects primarily to the basal half of the cochlea (Fig. 3), MOC effects may be restricted to high-frequencies, and thus to tasks in which interaural level differences or spectral cues are essential. Alternately, training animals after lesioning may have masked some lesion effects. Larger numbers or more extensive testing might also have revealed effects of the lesions. Nevertheless, the data suggest that the OC system is not vital for azimuthal sound localization of broadband sounds under normal circumstances.

\section{Olivocochlear control and auditory plasticity after hearing loss}

Unilateral earplugging caused an immediate and marked decrease in localization accuracy in all experimental groups, and in both hemifields. Decreased performance was always greatest for sounds ipsilateral to the plug, as seen in humans (Van Wanrooij and Van Opstal, 2007; Kumpik et al., 2010) and in other ferret studies (Kacelnik et al., 2006; Bajo et al., 2010; Nodal et al., 2010). The asymmetry between the responses to sounds from each hemifield could be due to spectral cues being more effective on the side contralateral to the plug (Gold and Knudsen, 1999; Van Wanrooij and Van Opstal, 2007). In any case, there were no

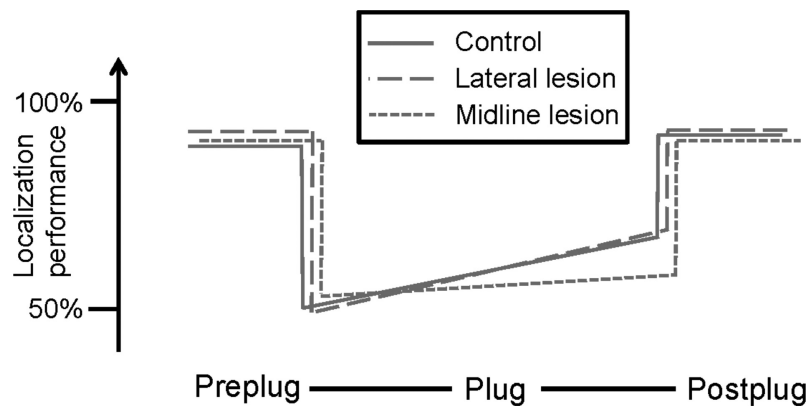

Figure 8. Schematic representation of the effects of $O C$ bundle lesions on sound localization and learning in ferrets. The vertical axes show the mean $\mathrm{CoM}(y)$ for a $1 \mathrm{~s} \mathrm{stimulus,} \mathrm{averaged} \mathrm{for}$ left and right earplugs. Lesions affected only the ability to relearn how to localize with altered binaural cues.

consistent differences in the initial plug-induced deficit among our different groups, regardless of the cues immediately available to perform the task, and consistent with the finding that OC lesions have little effect on normal sound localization.

Training in the localization task with the earplug in place led to improved localization for the control and lateral lesion groups, but not for the midline lesion group (Fig. 8). A similar disruption of localization improvement during earplugging was seen after severing the descending tracts from cortex to inferior colliculus (Bajo et al., 2010), and after lesions of the auditory cortex (Nodal et al., 2010).Thus, it appears that top-down communication from cortex to inner ear is required to refine localization abilities after hearing loss.

It seems that longer term learning has little effect on the response of the auditory system to earplugging, since rapid or immediate recovery of normal localization following unplugging has now been reported in several studies (Kacelnik et al., 2006; Kumpik et al., 2010) (S. Irving, A. Q. Sumnerfield, and D. R. Moore, unpublished observations). We did not observe any systematic biases in localization after removal of the earplug. However, small biases (after-effects) have been observed in the first session after plug removal in normal ferrets (Kacelnik et al., 2006). These after-effects may reflect longer term learning, although they were small and transient. Subsequent replugging resulted in reduced initial deficits and more complete adaptation. Earplug relearning thus appears to be rapidly reversible but, to some extent, remembered. It may be that animals develop multiple central representations of auditory space, maintaining normal "maps" as the default.

In our ferrets, the midline lesions symmetrically reduced both MOC and LOC projections in the two ears, while the lateral lesions reduced both LOC and MOC systems to the ipsilateral ear by up to $100 \%$. Thus, a moderate bilateral loss of OC feedback is more disruptive to localization plasticity than a more severe, but unilateral, loss of OC function. Given that both lesion types caused degeneration in LOC and MOC systems, it is impossible to conclude which of the two OC subsystems is more important for this plasticity. Indeed, both systems have the peripheral projection patterns to allow frequency-specific modulation of cochlear output in one or both ears through which the trainingrelated improvement could conceivably be effected.

One possibility, consistent with the abolition of learning only following midline lesions, is that OC influences can act flexibly through modulation of the input from just one ear. A lateral lesion leaves the OC inputs to one ear entirely intact, while a midline lesion disrupts the crossed fibers associated with both 
cochleae. Even in the case of a lateral lesion with a plug in the contralateral ear, the removal of all OC influences from an unplugged ear may be less detrimental than partial bilateral removal, as with a midline lesion. Note also that, in this scenario, interaural cues reintroduced on unplugging might be relatively unaffected even if some modulation persists after plug removal.

A recent human study (de Boer and Thornton, 2008) found an association between training-related improvement in a soundin-noise discrimination task and an increase in MOC reflex activity (measured by contralateral noise suppression of ipsilateral otoacoustic emissions), suggesting that plasticity in the MOC system can have a role in perceptual learning. Ipsilaterally evoked MOC activity was not assessed and there is, at present, no noninvasive test of LOC activity. Thus, it was not clear in this human study whether other aspects of OC system function were also altered as auditory performance improved.

According to current theory, effective use of binaural cues for precise azimuthal sound localization requires that sounds on the midline produce identical responses in the two ears. In the absence of experience-driven feedback control somewhere in the neural circuitry, this will be the case only if threshold sensitivities are precisely matched in the two ears, which they usually are not (Moore, 1993). Clearly, interaural symmetry is compromised by hearing loss. The present results suggest that one function of the olivocochlear neurons is to serve as the effectors in this top-down feedback system.

\section{References}

Bajo VM, Nodal FR, Moore DR, King AJ (2010) The descending corticocollicular pathway mediates learning-induced auditory plasticity. Nat Neurosci 13:253-260.

Brown MC (1987) Morphology of labeled efferent fibers in the guinea pig cochlea. J Comp Neurol 260:605-618.

Darrow KN, Maison SF, Liberman MC (2006) Cochlear efferent feedback balances interaural sensitivity. Nat Neurosci 9:1474-1476.

de Boer J, Thornton AR (2008) Neural correlates of perceptual learning in the auditory brainstem: efferent activity predicts and reflects improvement at a speech-in-noise discrimination task. J Neurosci 28:4929-4937.

Edmondson-Jones AM, Irving S, Moore DR, Hall DA (2010) A novel application of a centre of mass approach to the statistical analysis of localisation performance. Hear Res 267:4-11.

Gold JI, Knudsen EI (1999) Hearing impairment induces frequency-specific adjustments in auditory spatial tuning in the optic tectum of young owls. J Neurophysiol 82:2197-2209.

Groff JA, Liberman MC (2003) Modulation of cochlear afferent response by the lateral olivocochlear system: activation via electrical stimulation of the inferior colliculus. J Neurophysiol 90:3178-3200.

Guinan JJ Jr (1996) The physiology of olivocochlear efferents. In: The Springer handbook of auditory research: the cochlea, vol. VIII (Dallos PJ, Popper AN, Fay RR, eds). New York: Springer.

Guinan JJ Jr (2006) Olivocochlear efferents: anatomy, physiology, function, and the measurement of efferent effects in humans. Ear Hear 27:589-607.

Hine JE, Martin RL, Moore DR (1994) Free-field binaural unmasking in ferrets. Behav Neurosci 108:196-205.

Kacelnik O, Nodal FR, Parsons CH, King AJ (2006) Training-induced plasticity of auditory localization in adult mammals. PLoS Biol 4:e71.
King AJ, Parsons CH, Moore DR (2000) Plasticity in the neural coding of auditory space in the mammalian brain. Proc Natl Acad Sci U S A 97:11821-11828.

King AJ, Schnupp JW, Doubell TP (2001) The shape of ears to come: dynamic coding of auditory space. Trends Cogn Sci 5:261-270.

Knudsen EI, Esterly SD, du Lac S (1991) Stretched and upside-down maps of auditory space in the optic tectum of blind-reared owls: acoustic basis and behavioral correlates. J Neurosci 11:1727-1747.

Kumpik DP, Kacelnik O, King AJ (2010) Adaptive reweighting of auditory localization cues in response to chronic unilateral earplugging in humans. J Neurosci 30:4883-4894.

Liberman MC, Brown MC (1986) Physiology and anatomy of single olivocochlear neurons in the cat. Hear Res 24:17-36.

Liberman MC, Dodds LW, Pierce S (1990) Afferent and efferent innervation of the cat cochlea: quantitative analysis with light and electron microscopy. J Comp Neurol 301:443-460.

Liberman MC, Puria S, Guinan JJ Jr (1996) The ipsilaterally evoked olivocochlear reflex causes rapid adaptation of the $2 \mathrm{f} 1 \mathrm{-f} 2$ distortion product otoacoustic emission. J Acoust Soc Am 99:3572-3584.

Maison SF, Liberman MC (2000) Predicting vulnerability to acoustic injury with a non-invasive assay of olivocochlear reflex strength. J Neurosci 20:4701-4707.

May BJ (2010) Sound location: monaural cues and spectral cures for elevation. In: The Oxford handbook of auditory science: the Auditory brain (Rees A, Palmer AR, eds), pp 303-332. New York: Oxford UP.

May BJ, Budelis J, Niparko JK (2004) Behavioral studies of the olivocochlear efferent system: learning to listen in noise. Arch Otolaryngol Head Neck Surg 130:660-664.

Moore DR (1993) Plasticity of binaural hearing and some possible mechanisms following late-onset deprivation. J Am Acad Audiol 4:277-283.

Moore DR, Hine JE, Jiang ZD, Matsuda H, Parsons CH, King AJ (1999) Conductive hearing loss produces a reversible binaural hearing impairment. J Neurosci 19:8704-8711.

Nodal FR, Bajo VM, Parsons CH, Schnupp JW, King AJ (2008) Sound localization behavior in ferrets: comparison of acoustic orientation and approach-to-target responses. Neuroscience 154:397-408.

Nodal FR, Kacelnik O, Bajo VM, Bizley JK, Moore DR, King AJ (2010) Lesions of the auditory cortex impair azimuthal sound localization and its recalibration in ferrets. J Neurophysiol 103:1209-1225.

Rajan R (1990) Electrical stimulation of the inferior colliculus at low rates protects the cochlea from auditory desensitization. Hear Res 56:192-204.

Schofield, BR (2010) Structural organization of the descending auditory pathway. In: The Oxford handbook of auditory science: the auditory brain (Rees A, Palmer AR, eds), pp 43-64. New York: Oxford UP.

Van Wanrooij MM, Van Opstal AJ (2005) Relearning sound localization with a new ear. J Neurosci 25:5413-5424.

Van Wanrooij MM, Van Opstal AJ (2007) Sound localization under perturbed binaural hearing. J Neurophysiol 97:715-726.

Warr WB, Guinan JJ Jr (1979) Efferent innervation of the organ of Corti: two separate systems. Brain Res 173:152-155.

Winslow RL, Sachs MB (1987) Effect of electrical stimulation of the crossed olivocochlear bundle on auditory nerve response to tones in noise. J Neurophysiol 57:1002-1021.

Xiao Z, Suga N. (2002) Modulation of cochlear hair cells by the auditory cortex in the mustached bat. Nat Neurosci 5:57-63, 2002 Jan.

Yin TCT, Kuwada S (2010) Binaural localization cues. In: The Oxford handbook of auditory science: the auditory brain (Rees A, Palmer AR, eds), pp 271-302. New York: Oxford UP. 